tion than has been identified by other groups. The proportion of germline TP53 splicing mutations in LFS families may be markedly under-reported. We have examined the biological consequences of these mutations to determine the functional properties of the mutant p53 and to confirm that they are causative mutations. Previous studies have indicated that five main component tumors are associated with LFS: sarcomas, brain and breast tumors, leukemia and adrenocortical carcinoma. We have analyzed the observed incidence of cancers in first- and second-degree relatives of probands in comparison with that expected, on the basis of United Kingdom national cancer rates. The observed pattern of cancers in germline TP53 carriers was highly significantly different from that expected across all ages. Carcinoma of the breast, brain and spinal cord tumors, bone and soft-tissue sarcomas, adrenocortical carcinoma, Wilms tumor and malignant phyllodes tumors were all strongly associated with a germline TP53 mutation. Common carcinomas (such as lung, colorectal, bladder, prostate, cervix and ovarian) did not occur to excess. Germline TP53 mutations are associated with a subset of specific, mainly rare cancers.

Vidal, Marc

\section{The gene number dilemma: direct evidence for at least 19,000 protein-encoding genes in Caenorhabditis elegans and implications for the human genome}

David E. Hill, J. Reboul ${ }^{1}$, P. Vaglio ${ }^{1}$, N. Tzellas ${ }^{1}$, C. Jackson ${ }^{2}$ T. Moore $^{2}$, Y. Kohara ${ }^{3}$, J. Thierry-Mieg ${ }^{4}$, D. Thierry-Mieg ${ }^{4}$, J. Hitti $^{5}$, L. Doucette-Stamm ${ }^{5}$, J. Hartley ${ }^{6}$, G. Temple ${ }^{6}$, M. Brasch $^{6}$, D.E. Hill ${ }^{1} \& M$. Vidal ${ }^{1}$

${ }^{1}$ Dana-Farber Cancer Institute and Department of Genetics, Harvard Medical School, Boston, Massachusetts, USA

${ }^{2}$ Research Genetics, Huntsville, Alabama, USA

${ }^{3}$ Center for Genetic Resource Information, National Institute of Genetics, Shizuoka, Japan

${ }^{4} \mathrm{NCBI}$, NIH, Bethesda, Maryland, USA ${ }^{5}$ Genome Therapeutics Corp., Waltham, Massachusetts, USA

${ }^{6}$ Life Technologies Inc., Rockville, Maryland, USA

Estimates of the number of genes for organisms with a wide range of biological complexity will emerge from genome sequencing projects. This information will be crucial to correlate gene number with biological complexity. Computer-based annotations of the genome sequences of Saccharomyces cerevisiae, Caenorhabditis elegans and Drosophila melanogaster predict approximately 6,000, 19,000 and 13,600 genes, respectively. It is seemingly paradoxical that C. elegans contains $50 \%$ more genes than D. melanogaster and that the latter only contains twice as many genes as S. cerevisiae. However these numbers should be considered with caution until gene predictions are verified using experimental approaches. To experimentally verify gene predictions, we PCR amplified predicted open reading frames from a C. elegans cDNA library, cloned them and generated an ORF sequence tag (OST) for each of them. In a random sample $(n=1,200)$ of the $\sim 10,000$ genes predicted from the entire C. elegans genome but experimentally unverified so far, $90 \%$ of these predicted genes could be unambiguously verified by OST sequencing. When added to the list of $\sim 9,500$ genes previously verified by individual laboratories and/or by EST sequencing, our data confirm the existence of at least 19,000 protein-encoding genes in C. elegans. We suggest that ORFeome cloning coupled with OST analysis could be used to verify gene number predictions for other organisms. Furthermore, the demonstration and identification of 10,000 novel genes provides a resource for comparative genomics and gene expression studies. We also discuss our findings in the context of the current debate on the human gene number.
Villuendas, Raquel

\section{Expression microarrays in the prediction of treatment resistance: The CNIO program}

Raquel Villuendas, Jose Palacios, Manolo Morente, Orlando Dominguez, Javier Benitez \& M.A. Piris

Centro Nacional de Investigaciones Oncologicas, 28220 Madrid, Spain

The Molecular Pathology Program of the Spanish National Cancer Research Center (CNIO) performs molecular analyses of human tumors, allowing prediction of the treatment response to commonly used drugs and at the same time permitting the identification of relevant cancer genes. Biological therapy has so far been based on the study of unique genes or proteins, such as the administration of tamoxifen to endoplasmic reticulum positive tumors, Herceptin for cancer arising from overexpression of c-erb-B2, Cetuximab for cancers associated with overexpressed epidermal growth factor, Rituximab in CD20-positive lymphomas or RAR in progressive multifocal leukoencephalopathy. Although such biologically oriented treatment is making significant progress against different tumor types, it ignores the fact that cancer is a social and multigenic disease. The more common forms of cancer are the result of complex genetic alterations that allow the tumor cells to develop multiple capacities, such as tissue invasion and metastasis, angiogenesis promotion, unlimited replication, apoptosis escape, production of autonomous growth signals, insensitivity to cell cycle control signals and generation of genomic instability. One of the goals of the Molecular Pathology Program is to contribute to an individualized therapeutic strategy, according to the multiple and varied characteristics of the disease. The CNIO is analyzing resistance to commonly used drugs, through molecular study of targets and messenger RNA expression analysis using the microarray technology. The CNIO is also sponsoring the creation of a network of tumor banks in leading hospitals, which will allow massive application of these molecular techniques to large series of patients, diagnosed and treated using standardized protocols. Current projects include the following: (1) A study of the molecular mechanisms of therapeutic failure in cutaneous T-cell lymphoma and malignant melanoma treated with interferon- $\alpha$, PUVA (a combination of psoralen and longwave ultraviolet radiation) or both. The objective of this project is to identify the genes involved in interferon- $\alpha$ resistance in myelofibrosis and malignant melanoma. (2) Development of a complementary DNA biochip for the analysis of molecular changes associated with treatment resistance in breast cancer. The CNIO is open to establishing collaborations with other oncology groups to perform integrated analyses of the molecular factors predictive of treatment resistance.

Vinals, Carla

$[55]$

\section{Discovery of new potential antigens for cancer immunotherapy using "custom tissue" screening of a database of expressed sequence tags}

\section{Carla Vinals, Swann Gaulis, Jean-Pol Cassart \& Thierry Coche}

SmithKline Beecham Biologicals, 89 rue de l'Institut, 1330 Rixensart, Belgium

Immunotherapeutic approaches to fighting cancer are based on the principle of mounting an immune response (either an antibody response or a cytotoxic or $\mathrm{T}$ helper response) against a self-antigen expressed by the tumor cells. To reduce potential autoimmunity side effects, the antigens used should be as tumor-specific as possible. A complementary approach to experimental tumor antigen discovery is to screen the human genome in silico, particularly the databases of expressed sequence tags, in search of tumor-specific and tumor-associated antigens. We describe a new method of 'customized' screening of a database of expressed 